\title{
Routes migratoires et circulations en Chine : entre mobilités intracontinentales et transnationalisme
}

Laurence Roulleau-Berger et Lu Shi

\section{(2) OpenEdition \\ 1 Journals}

Édition électronique

URL : https://journals.openedition.org/remi/2012

DOI : 10.4000/remi.2012

ISSN : $1777-5418$

Éditeur

Université de Poitiers

\section{Édition imprimée}

Date de publication : 1 décembre 2004

Pagination : 7-27

ISBN : 2-911627-38-5

ISSN : 0765-0752

\section{Référence électronique}

Laurence Roulleau-Berger et Lu Shi, «Routes migratoires et circulations en Chine : entre mobilités intracontinentales et transnationalisme », Revue européenne des migrations internationales [En ligne], vol. 20 - n`3 | 2004, mis en ligne le 25 septembre 2008, consulté le 14 avril 2022. URL : http:// journals.openedition.org/remi/2012 ; DOI : https://doi.org/10.4000/remi.2012

Ce document a été généré automatiquement le 14 avril 2022.

(c) Université de Poitiers 


\title{
Routes migratoires et circulations en Chine : entre mobilités intracontinentales et transnationalisme
}

\author{
Laurence Roulleau-Berger et Lu Shi
}

Aujourd'hui, en Chine continentale, les migrations des populations des provinces chinoises vers les mégalopoles chinoises n'ont cessé de s'intensifier avec les processus de restructuration économique et de différenciation sociale dans une société de plus en plus inégalitaire. Ces populations ne cessent de se déplacer d'une province à l'autre pour constituer très souvent une main-d'œuvre très faiblement rémunérée sur les marchés du travail chinois et parmi elles certaines sortent de Chine pour venir en Europe de l'Est ou de l'Ouest sachant qu'à côté de ces « exclus de l'intérieur » de petits entrepreneurs chinois développent aussi des projets migratoires vers l'Europe. En effet depuis 1990, l'Europe de l'Ouest et plus récemment l'Europe de l'Est ont connu une augmentation sensible de leur immigration en provenance de la Chine continentale. Les flux d'immigration familiale continuent alors à prédominer et les migrations temporaires à des fins d'emploi ne cessent de prendre de l'importance. En ce qui concerne les migrants chinois, ils se dirigent encore principalement vers les États ayant une importante communauté chinoise (France, Grande-Bretagne, Pays-Bas, Allemagne) (Ma Mung, 2004 ; Benton et Pieke, 1998; Christiansen, 2003; Giese, 2003) mais ils ont investi aussi les «nouveaux " pays d'immigration de l'Europe du Sud (Italie, Espagne, Portugal...) (Beltran, 1997 ; Beltran et Saiz, 2001 ; Baganha, Marques et Gois, 2004 ; Ma Mung, 2002; Miranda, 2002) et depuis 1989, l'Europe de l'Est; les Chinois sont récemment arrivés en Bulgarie (Krasteva, 2004), en Russie (De Tinguy, 2003), en Tchéquie, (Moore, 2004), en Hongrie (Pieke, Nyiri, Thuno et Ceccagno, 2004) et en Yougoslavie qui sont devenus des espaces de transit et d'installation pour ces populations (Pina-Guerassimov, 2003). 
2 Les principales provinces d'émigration chinoise sont celles du Zhejiang et du Fujian, et plus récemment la Chine du Nord-Est, région où était implantée traditionnellement l'industrie lourde chinoise; en effet de plus en plus de licenciés(ées) des entreprises d'État de la Chine du Nord-Est, tendent à migrer vers l'Europe ; on peut d'ailleurs parler de nouvelle vague migratoire. Si la migration chinoise ne peut être considérée comme un phénomène nouveau en Europe de l'Ouest, si elle s'est constituée en Europe de l'Est au cours de ces quinze dernières années, ces migrations se sont intensifiées dans un contexte de transformations des marchés du travail nationaux avec les nouvelles politiques d'émigration en Chine et l'ouverture des pays d'Europe centrale et orientale. Les populations chinoises développent des stratégies migratoires de plus en plus sophistiquées sur une diversité de routes, elles ne fonctionnent pas dans un dispositif économique strictement localisé dans un cadre national mais dans des réseaux transnationaux diasporiques qui se caractérisent par la multipolarité de la migration et l'interpolarité des relations entre les différents lieux de fixation (Ma Mung, 2000). Les populations chinoises développent alors des «savoir-circuler» qui se traduisent par des mobilités intercontinentales et intracontinentales.

3 Nous proposons donc dans cet article de nous pencher plus précisément sur les migrations intracontinentales afin de comprendre comment se construisent les migrations transnationales, migrations liées entre elles par des réseaux économiques et sociaux fondés sur des relations de solidarité et d'entraide mutuelle. Nous nous intéresserons ici plus particulièrement aux mobilités, circulations et déplacements des migrants peu qualifiés, c'est-à-dire les paysans et les ouvriers-paysans qui quittent les campagnes chinoises, sachant que parmi ces paysans et ces ouvriers-paysans certains quitteront la Chine pour l'Europe où ils se retrouveront relégués dans des emplois très disqualifiants dans la restauration ou la construction pour les hommes, la confection, la restauration et parfois la prostitution pour les femmes. On regardera comment les espaces circulatoires en Chine continentale et les temporalités des déplacements ne cessent de se transformer, comment se diversifient des parcours, comment les destinations deviennent de plus en plus lointaines et comment se multiplient les lieux successifs d'installation à travers des formes migratoires où la polymigration intracontinentale apparaît de plus en plus marquée. On posera comme hypothèse que les opportunités structurelles, les modes d'inscription dans des réseaux sociaux, les stratégies individuelles et les "savoir-circuler» (Tarrius, 2000) participent ensemble à définir des formes différenciées de « capital spatial » (Lévy, Lussault, 2003) ${ }^{1}$ qui agissent sur le capital social, le capital économique et le capital symbolique pour définir une diversité de routes et parcours migratoires. Nous retiendrons que, dans un contexte de grande transformation, les deux sous-hypothèses de la force de la position et de la force des liens doivent être posées simultanément pour comprendre les stratégies et les parcours migratoires des migrants chinois qui développent des itinéraires de mobilité horizontale et verticale dans une société de plus en plus stratifiée et inégalitaire. 
Mise en place du dispositif méthodologique

Dans le cadre du programme de recherche "Migration, disqualification sociale et accès aux marchés du travail à Shanghai » qui a commencé en avril 2002 et co-financé par la DRI du CNRS, MIRA Rhône-Alpes et le Réseau des Maisons des Sciences de l'Homme (MSH) nous avons mis en place un dispositif méthodologique à trois volets :

- une ethnographie des quartiers de migrants à Shanghai

- une approche des marchés de l'emploi à Shanghai

- la réalisation des parcours biographiques de migrants

Ethnographie des quartiers de migrants à Shanghai

Nous avons commencé par une description ethnographique de la morphologie, de la structure, de l'organisation urbaine et économique des quartiers de migrants à Shanghai afin d'appréhender les processus de mise à distance des marchés du travail des populations migrantes en appréhendant les réseaux d'informations, d'échanges et de transactions qui les lient à leurs villages. À partir d'observations in situ sur les marchés de produits agricoles du quartier de Zhabei à Shanghai et d'investigations dans des rues de Zhabei, nous avons d'abord rencontré des migrants, des hommes et des femmes, originaires des provinces du Anhui, Jiangsu, Fujian, Jiangxi et Shandong qui ont entre 20 et 40 ans, faiblement dotés scolairement et dont la nature des activités professionnelles varie fortement.

Approche des marchés de l'emploi à Shanghai

Nous avons aussi analysé les dernières données statistiques du recensement national de 2000 et celles de la population migrante de Shanghai de 2002 pour situer la présence des migrants sur les secteurs des marchés du travail. Nous avons donc poursuivi nos investigations de terrain en tenant compte des taux de présence des migrants sur les différents segments de marché :

- sur des marchés plus ou moins formels des quartiers populaires

- dans des entreprises du bâtiment et de construction

- dans des entreprises de fabrication et de rénovation de meubles

- dans les nouveaux secteurs de services, notamment dans des salons de coiffure

- dans les secteurs des commerces et de la restauration

- dans le secteur de la grande distribution

Réalisation des parcours biographiques de provinciaux

Nous reconstituons les parcours biographiques des migrants en leur demandant leur formation, leur niveau scolaire, quelles ont été leurs expériences professionnelles dans leur ville ou village d'origine, leurs expériences de mobilité dans différentes provinces. Nous identifions toutes les situations de travail, les conditions de travail, la nature du contrat quand ils en ont, les relations de travail, avec la famille. Nous appréhendons les réseaux de solidarité et d'appartenance qui leur permettent d'accéder à des emplois urbains.

Nous avons déjà réalisé 140 entretiens biographiques avec ces provinciaux, souvent de jeunes hommes, qui arrivent d'abord du Anhui, du Jiangsu, du Henan, du Yunnan, du Gansu, de Chine du Nord à Shanghai et ensuite de toutes les autres provinces de la Chine continentale. 


\section{Migrations empêchées, migrations « ordonnées » et migrations « libres »}

4 La migration intracontinentale en Chine continentale peut être définie comme un processus externe et interne de transformation des territoires (Faret, 2003) où des éléments modifient des situations locales induisant des pratiques de mobilité et où d'autres les freinent, les bloquent. En outre, cette migration ne peut être abordée en Chine continentale comme un agrégat de pratiques individuelles, elle apparaît largement comme un construit sociétal qui résulte des stratégies d'acteurs politiques et économiques et de celles des migrants. La migration intracontinentale peut être vue alors comme le produit d'interactions politiques, économiques et sociales entre différents acteurs individuels et collectifs de la société chinoise, produit qui varie selon les périodes historiques. En effet pouvoir se déplacer en Chine continentale lorsqu'on est un paysan ou un ouvrier-paysan ne prend pas la même forme aujourd'hui qu'hier. Une nouvelle géographie des migrations internes en Chine continentale se dessine alors que le fait migratoire devient un élément majeur dans une économie nationale de marché qui s'appuie sur une réserve de main-d'œuvre peu qualifiée et dans la redynamisation de marchés locaux et régionaux.

\section{Migrations empêchées et captivité spatiale}

5 Avant 1979 on peut parler de "migrations empêchées». En effet le système de l'enregistrement de la résidence et de l'État-civil, sous le nom du hukou, avait été mis en place en 1958 dans un contexte de pénurie de céréales et de migrations vers les villes; la mise en place de cette politique anti-migratoire s'appuyait sur le contrôle de la mobilité géographique des individus en dehors de leur lieu d'enregistrement de résidence. Le hukou a longtemps constitué un dispositif panoptique (Foucault, 1975) qui a permis de catégoriser, d'ordonner et de surveiller les populations rurales vécues comme menaçantes pour l'ordre urbain; mais il faut préciser que le hukou, remis en place par des nouveaux acteurs politiques au lendemain de la prise du pouvoir des communistes chinois, reste un héritage de la Chine traditionnelle. Dans un souci de consolider le nouveau pouvoir, il a été repensé au début des années 1950 pour « mieux contrôler les éléments anticommunistes » dans un premier temps; ce nouveau système de hukou a été mis en place progressivement, d'abord dans les villes et ensuite dans la campagne. S'il est devenu un nouvel instrument de contrôle des populations pendant les premières années de la nouvelle République, il a déterminé les comportements, les attitudes et modes de vie des Chinois. À partir du Premier Plan quinquennal en 1955, l'économie est placée sous le contrôle direct de l'État; en 1958, dans un contexte de pénurie de céréales, pour empêcher le départ de la main-d'œuvre rurale vers les villes, l'État surveille strictement le changement de hukou, tout mouvement migratoire, de la campagne vers les villes, des petites villes vers les villes moyennes et grandes. En même temps, de nouvelles mesures ont été prises en faveur des Chinois de milieu urbain: garantir la consommation des céréales et des produits alimentaires de base, accéder aux emplois, bénéficier des avantages sociaux... Cette politique du hukou clivait ainsi la population chinoise en deux catégories selon le lieu de résidence, urbaine ou rurale, et la nature des emplois, agricole on non agricole. 


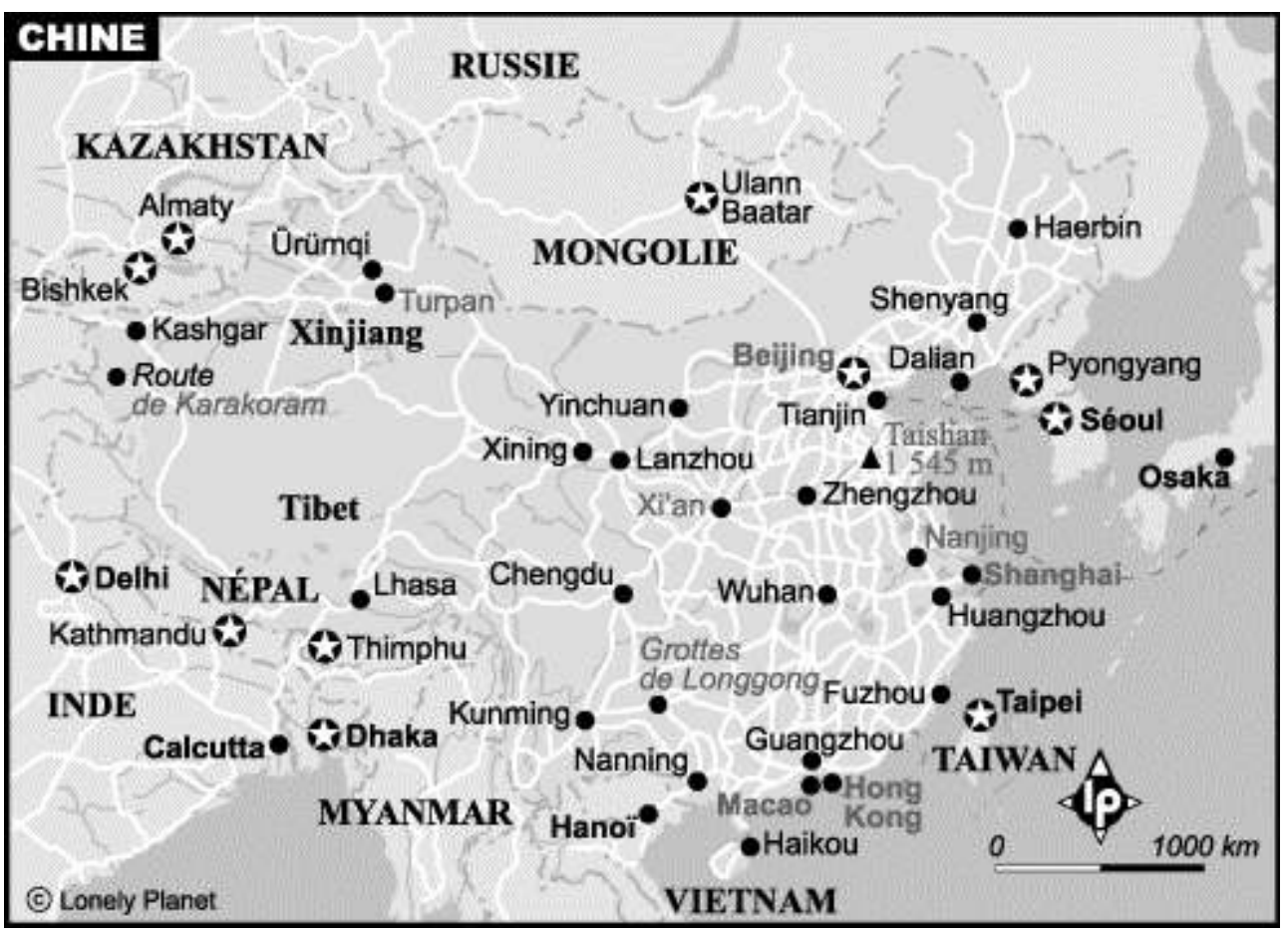

\section{Migrations ordonnées et mobilités limitées}

6 À partir des années 1960 jusqu'à la première moitié des années 1980 nous pouvons parler de migrations ordonnées; migrations qui prennent des formes différentes. En effet de 1961 à 1976, en même temps que les migrations empêchées, nous pouvons dire qu'il existe des migrations ordonnées voire même forcées des villes vers les campagnes; ce mouvement migratoire pourrait être défini comme un processus de contre-urbanisation. À la suite des trois années de famine (1959-1961), le gouvernement a décidé d'envoyer des citadins à la campagne afin de diminuer la croissance démographique urbaine et la pénurie alimentaire en ville. Ainsi, de 1961 à 1965, le taux de croissance de la population urbaine diminue de $4,4 \%$ chaque année. Ce mouvement migratoire ordonné sera suivi par une migration massive des « jeunes instruits » (zhishi qingnian) dans la campagne pendant la Révolution Culturelle. Dans ce mouvement " aller à la campagne et à la montagne " (Shangshan xiaxiang), 40 millions de jeunes citadins ont été concernés (Lu 2002, Wang 2003). Depuis la réforme économique de 1978, les migrations des campagnes vers les villes à partir de mobilités limitées ont de nouveau lieu. La Chine entre dans un processus d'urbanisation face à la "décollectivisation » des campagnes, l'excès de main-d'oeuvre rurale et le nouveau système de responsabilité familiale (jiating chengbao zhi): le gouvernement chinois redéfinit les politiques migratoires avec la suppression des restrictions de déplacement, l'instauration de rations alimentaires en ville et l'autorisation de résider en ville avec un certificat temporaire. L'abandon de l'agriculture collective conduit à une industrialisation dans les campagnes qui se traduit par de nombreuses créations d'entreprises rurales (xiangzhen qiye) dans les bourgs et cantons ; les paysans s'engagent dans des activités économiques, notamment industrielles, en restant dans leur province, voire leur canton. Le mouvement migratoire au début des années 1980 se 
caractérise par une mobilité intraprovinciale "quitter la terre sans quitter la campagne" (litu bu lixiang). Cette migration ordonnée a produit des mobilités géographiques limitées à des espaces restreints et fermés. Puis, les migrations internes en Chine continentale vont se diversifier dans un contexte de mutation politique et économique.

\section{Migrations organisées, migrations volontaires, mobilités intracontinentales et transnationales}

7 Depuis 1985 les mouvements migratoires ont pris de plus en plus d'ampleur en Chine continentale. Le système de hukou en tant que dispositif panoptique s'est affaibli et à favoriser des mobilités larges et ouvertes dans le cadre de "migrations volontaires » qui viennent concurrencer les migrations organisées et peuvent précéder des migrations transnationales.

8 Les migrations ont d'abord été organisées dans le cadre des politiques de lutte contre la pauvreté afin de favoriser la croissance des marchés du travail dans les villes et d'améliorer les conditions de vie dans les campagnes pauvres. Ces migrations organisées restent des dispositifs politiques de contrôle des mouvements migratoires vers les villes et "protègent " ainsi les marchés des emplois urbains. Les migrations organisées concernent les populations des régions Ouest de la Chine, et les groupes minoritaires des régions frontalières. Des acteurs publics et privés se trouvent impliqués dans la mise en œuvre de ces politiques; depuis 1989, la municipalité de Shanghai organise les migrations des jeunes originaires des provinces du Yunnan, du Shandong, Anhui et Jiangxi. Le directeur d'une agence intérimaire à Shanghai en témoigne :

«Dans le Yunnan, le nombre de personnes dont les revenus sont inférieurs à 600 yuans par an s'élève à 7 millions. Ici, leur salaire mensuel représente leur revenu annuel dans le Yunnan. Si les jeunes envoient 100 yuans par mois à leur famille, la famille peut manger à sa faim. Shanghai travaille avec quatre districts du Yunnan pour faire venir les jeunes; cette politique consiste à réduire les différences entre l'Est et l'Ouest de la Chine. Un jeune signe un contrat de 2 ans; après, il peut rentrer au village ou il reste à Shanghai. Depuis mars de cette année, je vais tous les mois dans le Yunnan pour recruter les jeunes. Ils viennent très nombreux, une centaine à chaque fois. Les jeunes sont encouragés aussi à venir par leurs propres moyens. Cette politique de la lutte contre la pauvreté a aidé le Yunnan à se développer. Les jeunes migrants envoient de l'argent chez eux. Cette province s'est beaucoup développée. Mon agence aide à la fois les jeunes de la région pauvre et les entreprises shanghaiennes. Je suis indépendant, mais sous contrôle du bureau de travail de la municipalité ».

9 Aujourd'hui les migrations organisées et les migrations volontaires se superposent. En 1985, l'État a décidé de baisser le prix d'achat des céréales afin de réduire les subventions à la consommation urbaine et les subventions d'aide aux entreprises rurales; cette situation a produit une main-d'œuvre excédentaire dans les campagnes chinoises. Par ailleurs, l'assouplissement du hukou a favorisé le départ de beaucoup de provinciaux en ville.

10 Enfin nous assistons dans les années 1990 à un mouvement migratoire intensif, dû notamment à la migration individuelle et volontaire qu'on peut qualifier de «larges ». Actuellement le nombre de migrants dans les villes chinoises s'élève à 120 millions, soit $10 \%$ de la population chinoise, alors qu'en 1992, ils étaient 46 millions. À Shanghai, 
selon le recensement de 2000 de la population migrante, le nombre total des provinciaux s'élève à 3 millions 871 100, leur présence dans cette ville a augmenté de 40,3\% par rapport à l'année 1997 (Roulleau-Berger et Shi, 2003). Ce sont les migrations « larges » qui peuvent être analysées comme des formes de mobilités pouvant anticiper des migrations transnationales.

\section{Diversification des formes migratoires en chine continentale et multipolarité des territoires circulatoires}

11 En expérimentant les migrations empêchées puis les migrations ordonnées et enfin volontaires, le migrant chinois s'est progressivement construit comme acteur capable d'action et de stratégies dans un contexte autoritaire. Les migrants que nous avons rencontrés disposent de faibles ressources individuelles - acquises ou héritées sociales, économiques et symboliques. Mais leur savoir-circuler sur le continent chinois n'apparaît pas systématiquement lié à la faiblesse ou à la force du capital social. Leur capital spatial se construit dans la capacité à articuler différentes métriques. Il se transforme en fonction des obligations de réciprocité construites à partir des solidarités familiales ou de solidarités villageoises. Il évolue avec les situations d'activité économique auxquelles les migrants accèdent et leurs capacités à cumuler les bénéfices selon les potentialités des lieux. Mais les liens forts et les liens faibles se conjuguent pour transformer le capital spatial, social et économique des migrants chinois. On peut distinguer plusieurs formes migratoires qui dépendant des modes d'agencement entre espaces, savoir-circuler et activités économiques différenciées : les migrations pendulaires, les polymigrations intracontinentales et les migrations entrepreneunariales. On peut faire l'hypothèse que les polymigrations intracontinentales et $a$ fortiori les migrations entrepreneuriales peuvent précéder les migrations internationales. 
Carte 2 : Les limites de Shanghai

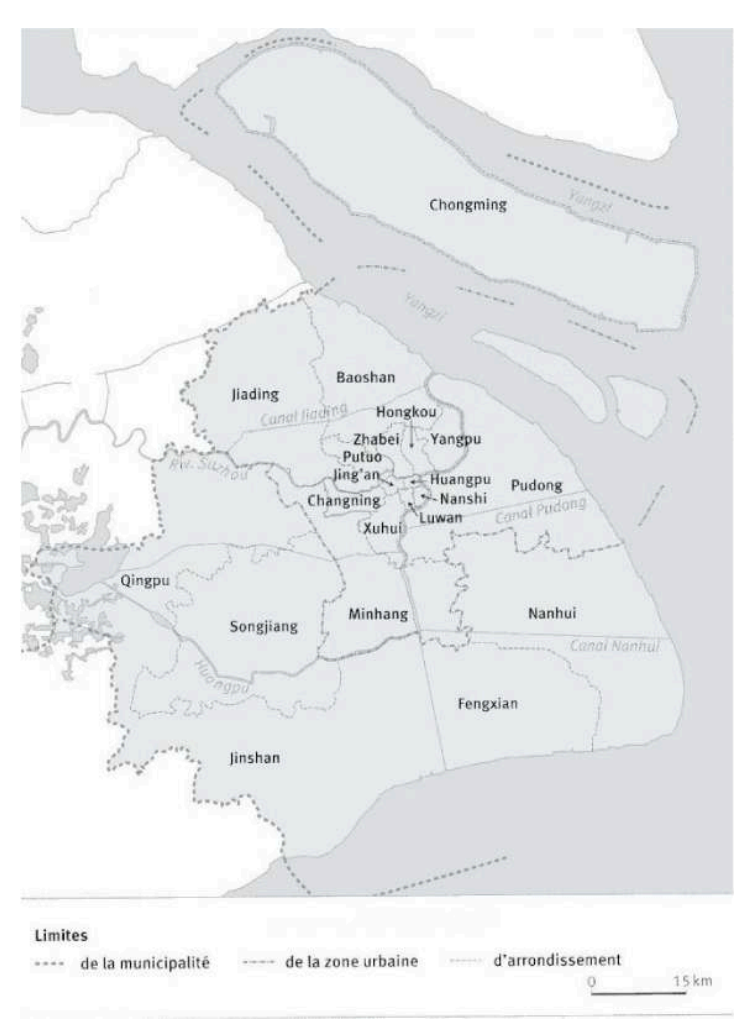

\section{Migrations pendulaires et monoactivité économique} différentes. Les migrations pendulaires caractérisent des populations chinoises faiblement qualifiées, disposant de peu de réseaux sociaux qui leur permettent cependant de quitter les campagnes mais dont les ressources sociales, économiques et symboliques sont faibles. Dans les migrations pendulaires les migrants développent des savoir-circuler entre deux lieux ou deux espaces liés à des réseaux familiaux et villageois restreints. Leurs pratiques économiques ne sont organisées qu'autour d'une même activité. Les migrations pendulaires peuvent relier une province à une autre ou bien s'organiser à l'intérieur d'une même province. Elles peuvent être hebdomadaires, saisonnières ou régulières. Les migrations pendulaires saisonnières peuvent être illustrées par le cas de ces migrants qui travaillent sur des chantiers de construction et qui repartent selon les saisons travailler aux champs dans les villages. Un autre type de situation rencontrée est celle de ces ouvriers employés sur un chantier de décoration d'appartements qui viennent presque tous du même district du Jiangsu et qui s'organisent au moment des récoltes pour rentrer au village à tour de rôle au moins une fois par an pour aider leurs familles aux travaux des champs.

Les migrations pendulaires concernent plus particulièrement les femmes. Les rythmes migratoires sont en effet plus soutenus pour celles qui retournent voir leurs enfants dans les villages plus fréquemment que les hommes. Ici, les opportunités structurelles apparaissent faibles, le volume de capital spatial reste constant et n'agit pas sur le volume de capital social qui reste faible et se maintient comme tel. Les migrations pendulaires évoluent rarement vers des migrations transnationales.

Revue européenne des migrations internationales, vol. 20 - n³ | 2004 


\section{Polymigration intracontinentale et pluriactivité économique} polymigration intracontinentale lié au petit commerce, une polymigration intracontinentale aléatoire, une polymigration intracontinentale liée à des activités artisanales. l'agencement de plusieurs espaces liés à des territoires géographiques éloignés et reliés entre eux par la capacité à faire circuler des biens matériels dans le cadre d'activités économiques comme le commerce de gingembre, de champignons, de graines, de jade, de grillons, de cuir, de vannerie, de piles... La faible valeur de ces biens matériels est associée à des qualifications techniques et professionnelles faibles et à des savoir-faire improvisés qui s'inventent sur les routes migratoires comme par exemple ces ouvriers licenciés d'entreprises d'État de la Chine du Nord-est qui se reconvertissent dans le commerce de grillons entre autres.

«Mon mari était chauffeur dans un établissement administratif dans le Heilongjiang. Mais, il a été licencié pour des raisons économiques. Moi, je ne travaillais pas non plus. À l'époque, notre fille a commencé à grandir, nous avions besoin d'argent. Dans le Nord-Est, on ne trouve pas de travail en ville et donc, nous avons décidé de partir. Nous voulions voir si nous pourrions mieux vivre ailleurs. Nous pensions qu'à Shanghai, il était beaucoup plus facile de gagner de l'argent qu'à Heilongjiang. Nous sommes venus le $1^{\text {er }}$ janvier 1999. Au début, nous vendions des produits alimentaires, mais ce n'était pas rentable. Nous allions chercher nos marchandises au Nord-Est par le train et nous revenions en bateau, cela faisait très loin. Et après, nous avons commencé à vendre des plantes, nous allions chercher des plantes dans notre région. Maintenant, en été, les plantes ne se vendent pas très bien, donc, nous vendons aussi des grillons. Les Shanghaiens aiment bien les grillons. Quand les affaires marchent bien, nous pouvons gagner plus de mille yuans par mois et à la saison morte nous pouvons manger à notre faim ».

Cette forme de polymigration intracontinentale lié au petit commerce se construit sur des lignes de réseaux de production et de circulation de denrées et produits divers où des petits commerçants, en prenant appui sur des solidarités familiales ou de villages, développent des savoir-circuler qui leur donnent accès à une diversité de destinations où ils développent des compétences de négociation sur des marchés ruraux et urbains 
pour y gagner une place (Roulleau-Berger et Shi, 2004). Les ressources individuelles jouent ici aussi fortement que les ressources collectives dans l'augmentation du capital spatial, du capital social et économique.

- La polymigration intracontinentale aléatoire se construit à partir de l'agencement de plusieurs espaces liés à des territoires géographiques éloignés et reliés entre eux par la capacité à passer d'une activité économique à une autre en développant des compétences à la fois adaptatives et techniques dans des emplois très faiblement qualifiés et radicalement différents. Le parcours de Huang Bei montre comment, à l'âge de 25 ans, sans qualification professionnelle, elle a déjà parcouru le Hubei, le Yunnan, le Guangdong et Shanghai où elle a accédé à chaque fois à des emplois dont les contenus diffèrent totalement.

Huang Bei quitte le Hubei à 17 ans pour aller aider son oncle dans le Yunnan à vendre des vêtements de militaires pour les migrants travaillant sur les chantiers. Son oncle part en Birmanie, elle reste un peu plus de deux ans dans le Yunnan où elle tient le magasin. Mais les affaires ne marchent pas bien, elle rentre alors au village. Quelques mois après, elle part avec des copines de son village dans le Guangdong pour travailler dans une usine de chaussures de cuir. Mais elle fait des allergies au cuir, elle doit donc quitter cet emploi, elle trouve un autre travail dans une usine électronique. Puis, par l'intermédiaire de sa soeur elle accède à un emploi de serveuse dans un restaurant; mais ce travail ne lui convient pas, les conditions sont très dures, elle rentre une deuxième fois au village ; ses parents ne veulent pas qu'elle reparte du fait qu'elle est en âge de se marier mais elle décide peu de temps après d'aller à Shanghai en 2003. Elle travaille 8 heures par jour dans un café situé dans le quartier de Zhabei, son salaire actuel est très bas et elle ne supporte pas très bien ce travail.

Ces populations de migrants, majoritairement des jeunes qui ont entre 16 et 30 ans, des garçons et des filles, développent des parcours de polymigration intracontinentale aléatoire qui se construisent dans la diversité de lieux successifs d'installation éloignés les uns des autres où se présentent des opportunités d'emploi ; ces lieux feront l'objet d'appropriations différenciées en fonction des aspirations individuelles mais surtout des conditions de travail imposées, souvent très dures. Ici on peut parler d'une capacité d'articuler les différents lieux entre eux mais pas de capacités d'accumulation de nouvelles ressources.

- La polymigration intracontinentale liée à des activités artisanales qui se construit à partir de l'agencement de plusieurs espaces liés à des territoires géographiques éloignés et reliés entre eux par la capacité à passer d'une activité économique à une autre en mobilisant des savoir-faire professionnels acquis dans les villages d'origine (maçonnerie, travail du bois, peinture, céramique...). Les ressources individuelles et les ressources collectives jouent ensemble un rôle décisif dans le déroulement des parcours migratoires. Des migrants vont mobiliser de manière cumulative des savoir-faire ouvriers ou artisanaux acquis dans des situations de travail variées où sont inégalement reconnues et légitimées les qualifications et compétences techniques.

«Je sais faire de la peinture, de la menuiserie, de la maçonnerie, je sais aussi travailler la tige d'acier, j'ai tout appris par moi-même, je suis un maître dans mon village où j'ai formé des dizaines d'apprentis. J'ai appris le métier de menuisier au village, c'est une tradition chez nous. Je n'ai pas terminé le collège, mais, quand je vois des meubles, je comprends tout de suite comment les fabriquer. Aujourd'hui, je fabrique seulement des pièces qui demandent plus de technique. Je suis allé dans une dizaine de provinces en Chine. Au début, je suis allé à Changzhou, j'ai travaillé comme menuisier. Et après, en 1979, je suis arrivé à Nanjing. J'ai aussi travaillé à 
Beijing pendant plus d'un an, j'avais fait la connaissance d'un patron qui travaillait sur un chantier de construction à Beijing; en fait, c'était un entrepreneur très célèbre qui avait des affaires partout en Chine, même au Japon. Je suis allé à Beijing avec lui pour construire un immeuble de sept étages. En 1980, j'ai travaillé comme ouvrier dans une usine de chimie. Ensuite, je suis allé à Xian et j'y suis resté quelques années. En 1994, je suis arrivé dans le Nord-Est. En 1995, j'ai été appelé par le patron pour qui j'avais travaillé à Beijing pour rénover la statue de Bouddha à la Montagne Jiuhua. Après ce travail, je suis parti pour Shenzhen. Et après, comme mes enfants devaient passer des examens d'entrée au lycée, je suis retourné au village, et j'y suis resté 2 ans, j'ai construit trois fois ma maison moi-même. Au début, c'était un appartement de trois pièces et maintenant c'est une grande maison. Et après, je suis venu à Shanghai. Je compte travailler au moins encore dix ans, parce que mes enfants sont grands et il faudra les marier ».

On peut ici parler d'une polymigration intracontinentale liée à des activités artisanales où les migrants développent de fortes capacités d'articuler des lieux, des espaces et des activités économiques qui font augmenter le volume du capital spatial, social et économique. Cette capacité d'articuler des espaces circulatoires différents peut être mobilisée dans le cadre de migrations transnationales.

\section{Migrations et dynamiques entrepreunariales en Chine et à l'étranger}

Dans les migrations qui se construisent à partir de dynamiques entrepreunariales se développent de très fortes capacités d'articuler plusieurs lieux, territoires et réseaux économiques à partir de savoir-circuler et de fortes compétences à accumuler des ressources économiques, sociales et symboliques dans les différents espaces en suivant des filières de distribution et de diffusion de produits ou de création de services autour de la même activité économique. Différentes catégories d'entrepreneurs émergent dans différents secteurs industriels, le secteur de la restauration ou le commerce du cuir. Ici le volume de capital spatial ne cesse d'augmenter en agissant simultanément sur le volume du capital économique et du capital social toujours en hausse avec l'élargissement des répertoires de ressources individuelles et collectives dans une dynamique entrepreneuariale. En effet, les ressources collectives liées à l'appartenance à un même village ou à une même ville créent des chaînes de confiance qui assurent les conditions de succès de l'activité économique dans un cadre entrepreunarial le plus souvent familial.

« Je suis originaire de Wenzhou. Au début, j'ai fait du commerce de quincaillerie à Wenzhou. Et après, j'ai suivi mon mari dans le Nord-Est de la Chine; nous avons ouvert un magasin de quincaillerie, les affaires ont bien marché. Ce genre de magasin n'existait pas avant, nous étions les premiers. Et les gens du Nord-Est ont suivi notre exemple; beaucoup de magasins du même genre sont apparus. Nous avons eu l'idée de créer une société avec un partenaire de Tianjin pour développer nos affaires dans d'autres provinces. Mon mari a décidé ainsi de venir à Shanghai, il a ouvert une usine pour fabriquer des pièces électriques et il les vend dans toute la Chine. Il veut aussi les vendre à l'étranger. Nous sommes maintenant un groupe et mon mari embauche 300 employés dans l'usine de Shanghai. Nous avons des collaborateurs partout. Au début des années quatre-vingt, à Wenzhou, les gens fabriquaient beaucoup pour les marques japonaises. Maintenant, nous fabriquons pour nos propres marques. Les gens du Wenzhou sont intelligents, ils ont le sens du commerce. Je ne travaille pas pour mon mari. L'année dernière, une amie a cédé ce petit magasin de lingerie et je l'ai pris. Je ne veux pas rester sans rien faire. Ce magasin est mon passe-temps. Je cherche des modèles moi-même et ça marche bien jusqu'à maintenant ". 
Dans les différentes formes de migration intracontinentale se forment des «territoires circulatoires » (Tarrius, 2002) individuels et collectifs à échelles multiples et emboîtées qui combinent une pluralité de ressources et de modes de mobilisation de celles-ci. Mais, selon les formes de migrations, le volume du capital spatial augmentera plus ou moins. Dans la polymigration intracontinentale aléatoire et les migrations pendulaires, le volume du capital spatial tend à se maintenir du fait de compétences d'agencement d'espaces et de savoir-circuler, mais des difficultés de capitalisation d'expériences de travail de nature trop différente existent pour devenir cumulables. Dans la polymigration intracontinentale liée à des activités artisanales ou de petit commerce et les activités entrepreneunariales, le volume du capital spatial augmente du fait des fortes capacités d'articuler des lieux et des réseaux et d'y accumuler des ressources nouvelles dans le cadre d'activités économiques de la même nature en Chine ou à l'étranger.

Le premier restaurant Dongbeiren, monté par des Chinois originaires du Nord-Est a
été ouvert en 1993 à Hainan pour la clientèle originaire du Nord-est qui appréciait
la cuisine typique familiale du Nord-est avec les produits naturels de leur région.
Dans cette petite entreprise les employés veulent promouvoir la culture de la Chine
du Nord-Est. Ce petit restaurant familial est devenu en une dizaine d'années une
véritable entreprise ; aujourd'hui, on compte 14 restaurants dans le Sud de la Chine.
Les propriétaires possèdent leurs propres serres dans le Nord-est où ils
s'approvisionnent. Tous les jours, les légumes et les produits régionaux sont
transportés par avion du Nord-est au Sud de la Chine. Les propriétaires possèdent
aussi une usine à Canton pour fabriquer des blouses de style populaire du Nord-est
et des objets de décoration pour les restaurants. Cette chaîne de restauration ne
recrute que des jeunes du Nord-est, principalement les jeunes de la campagne. Les
jeunes sont logés et nourris sur place. L'entreprise organise par ailleurs des
cérémonies de mariage, des lunes de miel et des soirées d'anniversaire afin de
fidéliser le personnel. Les employés passent régulièrement des examens pour
augmenter la productivité et aussi des évaluations pour toucher la prime. La société
fournit des formations de comptabilité, de gestion et d'anglais afin de permettre au
personnel de monter en grade. Les mutations d'un restaurant à l'autre (il s'agit
souvent du changement de ville) sont gérées par un service du personnel qui
cherche à concilier l'offre de travail et la demande des employés. Ces derniers
peuvent aussi recommander leurs amis et proches du village pour les faire entrer.
L'année prochaine un nouveau restaurant sera créé à Beijing et un aux États-Unis.

24 Si les migrations pendulaires ne paraissent donc pas pouvoir évoluer vers des migrations internationales, on peut ici considérer que la polymigration intracontinentale ne pourra évoluer vers une migration internationale qu'à partir du moment où le volume du capital spatial, social et économique atteint un certain seuil. Mais c'est évidemment la migration entrepreunariale qui apparaît comme une forme migratoire anticipant le plus nettement la migration transnationale.

\section{Migrations, mobilité et reconnaissance sociale}

Il faut d'emblée préciser qu'avant de parler de mobilité horizontale ou de mobilité verticale, nous nous situons en Chine continentale dans un contexte de mobilité structurelle lié au processus de stratification sociale toujours plus marqué, à l'affaiblissement du monde rural et à l'exacerbation du processus d'urbanisation, voire de métropolisation; quand les paysans migrants sont contraints de quitter leurs villages pour aller travailler comme ouvriers en milieu urbain, ce qui pourrait 
apparaître comme une mobilité verticale ne peut l'être. Il s'agit plutôt de mobilité horizontale. Les transformations économiques, sociales et politiques en Chine continentale participent à intensifier et complexifier des mouvements migratoires qui permettent l'accumulation ou non d'un capital spatial susceptible d'agir sur les capitaux sociaux et économiques et produire alors des formes de mobilité sociale.

\section{Mobilité horizontale et stratégies de survie économique}

Les migrations pendulaires produisent des parcours de mobilité sociale horizontale c'est-à-dire des changements de position qui ne correspondent ni à une véritable promotion ni à une rétrogradation, comme pour ces ouvriers-paysans qui travaillent sur les chantiers de construction. Mais les parcours de polymigration intracontinentale aléatoire contribuent aussi à produire des carrières de mobilité horizontale.

« Je viens de Lanzhou, du Gansu. Il n'y a pas beaucoup d'argent, en plus il faut payer les différentes taxes. Sinon, ils viennent vous embêter. Dans cette région on plante des raisins et on les vend à Shanghai pour 2 yuans la livre. Je n'aimais pas les études ni le travail aux champs. J'étais très turbulent, je suis parti après le collège dans le Shanxi pour apprendre la cuisine dans une école pendant trois mois où j'ai été envoyé à la fin de mes études à Shenyang, nous étions trois à être envoyés là-bas. Nous travaillions pour un patron du matin au soir. Nous faisions tout, le travail de restaurant et aussi tous les travaux chez lui. Sa maison devait être démolie, alors nous avons travaillé très dur. Il fallait transporter des poutres sur les épaules; le soir, nous mangions beaucoup, beaucoup, c'était vraiment épuisant. J'avais 16 ans, les deux autres copains avaient 23 et 21 ans, j'étais le plus jeune. Le soir on dormait sur les bancs dans le restaurant, il faisait très froid. J'ai travaillé pour ce patron pendant un mois à peu près, j'en ai beaucoup bavé. Je raconte rarement mon histoire aux gens; après le premier mois, le patron voulait licencier deux personnes, j'ai été licencié. Le patron nous a présenté à un autre patron de restaurant, j'ai fait seulement quelques jours dans ce restaurant parce qu'il y a eu un meurtre, j'ai eu très peur et je suis parti. Je suis retourné voir l'ancien patron pour lui demander de me trouver un autre boulot. Je voulais simplement gagner quelques dizaines de yuans pour rentrer chez moi, il m'a fait entrer dans une usine de transformation de boissons. C'est une "usine noire", c'est-à-dire qu'on y entre et on ne peut plus ressortir; il y a un gardien à l'entrée qui nous surveille et nous interdit de sortir. Moi, je transportais chaque jour des caisses de boissons très lourdes, il fallait monter par un petit escalier pour les vider, c'était un escalier très étroit. Si on se cognait, le contremaître nous frappaient ; à la chaîne c'était pareil, si on travaillait moins vite, le contremaître frappait. Dans cette usine, il y avait pas mal de jeunes filles. Elles m'aidaient souvent, parce qu'elles devaient penser que j'étais trop jeune, ce qui a provoqué la jalousie chez les autres garçons. Un jour, je travaillais dans la chambre froide, ils m'ont enfermé dedans pendant plusieurs heures, j'avais les lèvres violettes, je me sentais mourir. Dans cette usine, on travaillait 24 heures sur 24, sans arrêt sauf une heure pour le déjeuner et une heure pour le dîner. On ne pouvait même pas dormir; il m'arrivait souvent de dormir debout. Du coup, je suis venu travailler dans cette usine avec l'autre copain. Quelquefois, je voulais laver des fruits, c'était moins fatigant, mais le contremaître arrivait tout de suite pour m'insulter et me demander de faire un travail très dur. Un jour, on m'a demandé de jeter des boissons périmées en dehors de l'usine, j'ai saisi cette occasion, j'ai pris mes bagages et me suis sauvé... Je suis parti avec mon copain qui était venu du Shanxi avec moi. Je n'avais pas de sous, je ne connaissais pas les lieux. Nous sommes arrivés devant un arrêt de bus, ce copain m'a dit: "Attends-moi, je vais acheter un truc". Je l'ai vu courir et j'ai compris qu'il ne reviendrait jamais. Il m'a laissé tombé, beaucoup d'amis m’ont blessé. J’ai appelé ma sœur qui travaillait à Shanghai, elle avait un ami dont la famille vivait à Fushun, il 
est venu me chercher à la gare de Shenyang pour m'emmener chez lui. Sa mère m'a trouvé un travail dans un restaurant. Après être resté un certain temps chez elle, j'ai gagné un peu d'argent et je suis venu à Shanghai. Ma sœur m'a fait entrer dans ce métier de coiffure. Maintenant, mon grand frère est aussi dans la coiffure. Nous voudrions ouvrir un salon chez nous plus tard car il y a trop de concurrence à Shanghai, tout est trop cher. J'ai beaucoup appris entre 16 ans et 17 ans. Je n'ai plus confiance en personne et je n'ai confiance qu'en moi-même ».

Dans les carrières de mobilité horizontale apparaît ici la figure du hobo (Anderson, 1993) moderne, qui circule sur le continent chinois entre deux emplois ou deux activités pour échapper aux conditions très dures de vie en milieu rural. Dans ce mouvement d'intensification des migrations internes en Chine, de passage d'une économie socialiste à une économie de marché, de fermeture des entreprises d'État, s'est constituée une grande réserve d'hommes et de femmes qui traversent le continent à la recherche d'un travail. Le hobo chinois peut être un cordonnier, un tailleur, un maçon, un ébéniste, un peintre en bâtiment... Mais ce hobo peut aussi apparaître sous la forme d'un jeune ouvrier ou d'une jeune ouvrière employée dans des entreprises chinoises ou sino-étrangères où il peut vivre une forme d'esclavage moderne dans un contexte de production globalisée. La polymigration intracontinentale aléatoire produit des carrières de mobilité horizontale où ce migrant peu qualifié se trouve contraint de subir la non-reconnaissance publique et sociale, voire l'humiliation et la violence symbolique et parfois physique dans des conditions de travail très éprouvantes, provoquant la perte de soi.

Les stratégies migratoires de survie individuelles et collectives, la relation négative entre capital spatial et capital social participent ensemble à définir des mobilités horizontales qui se construisent dans une mobilisation minimale et discontinue de ressources personnelles et sociales. Ces carrières de mobilité horizontale se développent à partir de liens forts dans des relations de négociation basées principalement sur des solidarités familiales. Les migrants, du fait de leurs origines rurales ont intériorisé ici l'idée de ne pas être reconnu dans cette société de plus en plus inégalitaire et se comportent comme des acteurs dominés capables cependant de produire des armes du faible (De Certeau, 1980); mais ils apparaissent peu enclins à développer des parcours migratoires internationaux.

\section{Mobilité verticale ascendante et lutte pour la reconnaissance sociale}

29 Les formes de polymigration intracontinentale liées aux activités de commerce ou artisanales, les migrations liées à des dynamiques entrepreunariales peuvent être qualifiés de parcours de mobilité verticale qui se construisent dans une lutte pour la reconnaissance sociale. Les fortes stratégies migratoires individuelles et collectives, la relation d'influence réciproque positive entre capital spatial et capital social, les opportunités structurelles participent ensemble à définir ces mobilités verticales dans une société chinoise de plus en plus stratifiée. Apparaissent alors ici la figure du commerçant ou de l'artisan et celle de l'entrepreneur.

Nous avons rencontré de petits entrepreneurs dans les secteurs de l'insertion professionnelle avec la mise en place d'agences intermédiaires pour l'emploi, de nouveaux services urbains, de création de salons de coiffure, de commerces et de restaurants... comme ces migrants du Nord-est qui ont créé dans le Sud de la Chine une 
chaîne de restaurants spécialisés dans la gastronomie du Nord-est ou ces Wenzhou dans l'industrie du cuir qui jouent un rôle majeur dans son développement. Nous pouvons considérer ici que les entrepreneurs du Nord-est et les Wenzhou créent de véritables niches économiques où, parvenus au statut de patrons, ils embauchent des migrants originaires de leurs provinces qu'ils intègrent dans leurs réseaux économiques.

31 Ces entrepreneurs créent leurs règles, conventions et normes sur des segments de marché qu'ils investissent. Ils développent de nouveaux savoir-faire dans la création de nouvelles filières, déploient une grande capacité à construire des réseaux de coopération économique et à mobiliser des ressources diverses et de liens sociaux. Ici la dispersion géographique inhérente aux formes de polymigration intracontinentale liées aux activités de commerce, artisanales ou entrepreneunariales produit simultanément du capital spatial et du capital social dans la multiplolarité et l'interpolarité (Ma Mung, 2000). Ces itinéraires d'entrepreneurs peuvent alors être définis comme parcours de reconnaissance sociale et symbolique par l'accès à un statut qualifiant et au prestige social en Chine, voire à l'étranger.

Ces mobilités verticales se construisent dans l'accumulation continue de ressources personnelles et sociales et surtout dans leur mobilisation maximale. Les carrières de mobilité verticale se construisent dans la capacité à gérer des situations d'incertitude économique, à développer simultanément des liens forts et des liens faibles dans les échanges et tractations économiques fondées sur des relations de confiance, des obligations réciproques (Lin, 1995; Faist, 2000), et des solidarités liées aux appartenances. Ces mobilités verticales au niveau subjectif produisent des identités sociales qui se construisent dans une lutte pour la reconnaissance sociale, ici l'accès à un statut et à une position sociale supérieure à celle des parents dans une société en grande transformation où la réputation et le prestige apparaissent comme des biens symboliques fondamentaux à acquérir.

Si les mobilités verticales ascendantes peuvent se maintenir dans des migrations transnationales, elles peuvent aussi se retourner en mobilité descendante quand les expériences et compétences construites dans la migration intracontinentale s'effacent très brutalement dans l'épreuve de la non-reconnaissance de l'Autre, notamment pour les migrants peu qualifiés qui arrivent sans papiers en Europe de l'Ouest ${ }^{2}$ (RoulleauBerger, 2004).

\section{Mobilité verticale descendante et perte de reconnaissance sociale}

Toutes les formes de migration peuvent produire des mobilités verticales descendantes quand au cours des différentes étapes migratoires, la migration signifie avant tout perte de ressources économiques, sociales et identitaires mais aussi effacement puis déni des qualifications, expériences et compétences acquises dans une dispersion, une discontinuité et une disjonction des activités économiques. Ici les volumes de capital spatial et social, déjà faibles au départ, ne cessent de diminuer, sont de moins en moins mobilisés et deviennent parfois quasiment invisibles.

"J'ai été fonctionnaire dans l'administration locale du bourg. Je m'occupais du recensement et de la gestion des champs. En 1990, dans le Anhui, il y eu la réforme dans la fonction publique: les bourgs ont été fusionnés. Donc, il y a eu le licenciement des fonctionnaires. J'ai été renvoyé dans un village pour être instituteur non titulaire. En 1996, l'éducation nationale a commencé la réforme. Les enseignants non titulaires pouvaient être titularisés à condition d'avoir commencé 
en 1984, moi j'avais commencé en 1990 seulement. Donc, j'ai été de nouveau licencié. J'avais un parent qui travaillait à Shanghai dans la construction, j'ai pensé qu'il fallait essayer. Je suis donc venu. Je me suis inscrit dans une agence d'emploi, j'ai trouvé un travail dans une usine tout de suite. Je m'occupais des stocks dans l'entrepôt. Et après, cette usine a été fermée. En 2001, je suis venu ici et j'y suis resté jusqu'à aujourd'hui. Je travaille comme responsable de stock ». chômage et pauvreté, les mobilités verticales descendantes peuvent apparaître comme des séquences biographiques ou traduire un processus d'éviction des marchés du travail urbain.

\section{Conclusion}

Dans une Chine en grande transformation se multiplient des formes migratoires, et notamment des polymigrations intracontinentales, qui traduisent une diversité de stratégies de réponse à des situations de grande pauvreté. Les parcours migratoires de ces paysans et ouvriers-paysans chinois se construisent à partir de relations entre une diversité d'espaces et d'activités dans lesquels circulent et se déplacent des groupes, des familles et des individus avec leurs répertoires de ressources, leurs compétences et savoirs. Les variations des effets du capital spatial sur le volume des capitaux sociaux, économiques et symboliques révèlent l'espace comme ressource utilisée et mobilisée différemment selon les individus et les groupes sociaux ; mais elles font aussi valoir des formes spécifiques d'encastrement, « c'est-à-dire la combinaison entre, d'une part, des facteurs cognitifs et culturels qui règlent comme limites et ressources de l'action économique et, de l'autre, les réseaux de relations sociales des acteurs insérés dans des contextes locaux et politiques" (Mingione, 2004). Ces formes spécifiques d'encastrement se construisent ici à partir de politiques migratoires liées à un système socialiste de marchés du travail à la fois segmentés, multipolaires et globalisés, et de stratégies de migrants, faiblement dotés en ressources, capables de se déplacer, de circuler, oscillant entre la figure du hobo et celle de l'entrepreneur, mais toujours soumis au risque de perdre tout accès à une place. Les circulations migratoires en Chine continentale articulent à la fois des échelles, locale, régionale et intracontinentale dans une relation dynamique pour laisser émerger des acteurs individuels et collectifs très contraints politiquement mais capables de mouvement, d'action, de mobilisation et d'engagement dans des activités économiques plurielles sur des espaces multipolarisés en Chine et dans le monde. L'espace migratoire à la fois local et global joue ici un rôle décisif dans la façon dont se configurent, se déforment, se transforment les territoires productifs en Chine continentale et à l'échelle internationale.

\section{BIBLIOGRAPHIE}

ANDERSON Nels, postface de SCHWARTZ, Olivier (1993) Le Hobo. Sociologie du sans-abri, Paris, Nathan.

Revue européenne des migrations internationales, vol. 20 - $n^{\circ} 3$ | 2004 
BAGANHA Maria, MARQUES José et GOIS Pedro (2004) New waves : migration from eastern to southern Europe, Luso-American Foundation, Lisbon.

BELTRAN Joaquin (1997) Immigrés chinois en Espagne ou citoyens européens ? Revue Européenne des Migrations Internationales, 13 (2) : 63-79.

BELTRAN Joaquin (2003) Los ocho inmortales cruzan el mar. Chinos en Extremo.

BELTRAN Joaquin y SAIZ Amélia (2001) Els xinesos a Catalunya. Familia, educació i integració. Alta Fulla, Barcelona.

BENTON Gregor et PIEKE Frank (1998) The Chinese in Europe, Basingstoke, Macmillan.

BUREAU DES STATISTIQUES DE SHANGHAI (2001) Shanghai Renkou Fazhan Baogao (Rapport sur le développement de la population à Shanghai), Bureau des statistiques de Shanghai.

CAI Fang (2002) Zhongguo renkou yu laodongli wenti baogao (Rapport sur la population et les mains d'œuvre en Chine), Édition Documentation science sociale, Beijing, 372 p.

CHRISTIANSEN Flemming (2003) China town in Europe. An exploration of overseas Chinese identity in 1990s, London, Routledge Curzon, 2003.

DE CERTEAU Michel (1980) L'Invention du quotidien, 1. Arts de faire, Paris, Gallimard, Folio.

DE TINGUY Anne (2003) L'immigration chinoise en Russie : source de conflit ou nécessité, Migrations Société, Vol. 15, $\mathrm{n}^{\circ}$ 89, Septembre.

FAIST Thomas (2000) The volume and dynamics of international migration and transnational social spaces, Oxford: Oxford University Press.

FARET Laurent (2003) Les territoires de la mobilité. Migration et communautés transnationales entre le Mexique et les États-Unis, Paris : CNRS Éditions.

FOUCAULT Michel (1975) Surveiller et punir, Paris, Gallimard.

GIESE Karsten (2003) Les Chinois en Allemagne - une minorité ethnique en cours de transformation. Migrations Société, Vol. 15, nº 89, Septembre-Octobre : 47-63

GIESE Karsten (2003) New Chinese Migration To Germany : Historical Consistencies and New Patterns of Diversification within a Globalized Migration Regime. International Migration, 41 (3) : 155-185

GUERASSIMOF Carine (2003) La France, carrefour européen de la nouvelle migration chinoise, Migrations Société, Vol. 15, nº 89, Septembre-Octobre : 47-63

HENRIOT Christian et ZHENG Zu'an (1999) Atlas de Shanghai, espaces et représentations de 1849 à nos jours, Paris CNRS Éditions.

KE Lanjun et LI Hanlin (2001) Dushili de Cunmin (Les villageois en ville), Éd. Zhongyang bianji chubanshe, $355 \mathrm{pp}$.

LEVY Jacques et LUSSAULT Michel (2003) : Dictionnaire de la géographie, Belin, Paris.

LI Peilin (1996) Les réseaux et la position sociales des travailleurs migrants (Liudong mingong de shehui wangluo he shehui diwei), Études en sociologie, $\mathrm{n}^{\circ}$ 4, pp. 42-52.

LI Peilin (2003) Nongmingong-Zhongguo jincheng nonmingong de jingji shejui fenxi (Les ouvrierspaysans : Analyses socio-économiques sur les ouvriers-paysans dans les villes chinoises), Édition Documentation science sociale, Beijing, 298 p.

LI Peilin, ZHANG Yi et ZHAO Yandong (2000) Jiuye yu Zhidu bianqian (Job searching, Employmen and Institutional Change), Zhejiang renmin chubanshe, $345 \mathrm{p}$. 
LI Yining (2001) Zhongguo chengzhen jiuye yanjiu (Étude sur les emplois dans les villes chinoises), Zhongguo Jihua chubanshe, $321 \mathrm{p}$.

LIN Nan (1995) Les ressources sociales, Revue française de sociologie, XXXVI-4, pp. 685-705.

LU Yilong (2002) Le système de hukou en Chine depuis 1949 : structures et évolution (1949 nian hou de Zhongguo huji whidu : jiegouyu bianqian), Journal de l'Université de Beijing, n² 2, pp. 83-85.

MA MUNG Emmanuel (2002) Migratory and economic networks of the Chinese diaspora in southern Europe, in Tsun-Wu Chang and Shi-Yeoung Tang (ed.) Essays on ethnic Chinese abroad. Taiwan, Academia Sinica Press.

MA MUNG Emmanuel (2004) (à paraître) : Immigration and ethnic labour market, in Felicitas Hillmann and Ernst Spaan (Éd.) Asian Migration and Labour market Integration in Europe : Routledge.

MA MUNG Emmanuel (2000) La diaspora chinoise, géographie d'une migration, Paris, Orphys.

MINGIONE Enzo (2004) Encastrement in LA ROSA Michèle et LAVILLE Jean-Louis La sociologie économique européenne : une rencontre franco-italienne, Milan, Franco Angeli.

MOORE Marketa (2004) Challenging the structure : Chinese women who made their mark in Prague in K. E. Kuah (Éd.) Chinese women and their network capital Occidente. Edicions Bellaterra : Barcelona.

PIEKE Frank N., NYIRI Pal, THUNO Mette et CECCAGNO Antonella (2004) Transnational Chinese: Fujianese Migrants in Europe, Routledge, London.

POPULATION CENSUS OFFICE OF SHANGHAI (2002) Shanghaishi 2000 nian Renkou Pucha Ziliao-wailai liudong renkou pucha shuju (Tabulation On The 2000 Population Censes Of Shanghai Municipality), Beijing, China Statistics Press, 417 p.

ROULLEAU-BERGER Laurence (2004) Insertions segmentées, travail et discriminations des femmes migrantes et de leurs filles, in Femmes d'origine étrangère : travail, accès à l'emploi, discriminations de genre, La Documentation Française, Paris, 2004.

ROULLEAU-BERGER Laurence (2003) La Chine au seuil du XXI ${ }^{\mathrm{e}}$ siècle : état des lieux, Revue française de science politique, $\mathrm{n}^{\circ} 4$.

ROULLEAU-BERGER Laurence et SHI Lu (2003) Les provinciaux à Shanghai : formes d'inscriptions urbaines et économiques des migrants dans la ville, Les Annales de la recherche urbaine, $n^{\circ} 93, \mathrm{pp}$ 49-56.

ROULLEAU-BERGER Laurence et SHI Lu (2004) Inégalités, disqualification sociale et violences symboliques à Shanghai : l'accès à l'emploi urbain des provinciaux, Le Journal des anthropologues, Globalisation : effets de marché et migrations, $n^{\circ}$ 96-97.

TARRIUS Alain (2002) La mondialisation par le bas. Les nouveaux nomades de l'économie souterraine, Paris, Balland.

TARRIUS Alain en collaboration avec MISSAOUI Lamia (2000) Les nouveaux cosmopolitismes. Mobilités, identités, territoires, La Tour D’Aigues, Éditions de l'Aube.

WANG Haiguang (2003) Macro analyse sur le système de hukou et son évolution en Chine contemporaine (Dangdai Zhongguo huji zhidu xingcheng yu yange de hongguan fenxi), Étude sur l'Histoire du Parti Communiste chinois, $\mathrm{n}^{\circ}$ 4, 2003, pp.41-43.

WANG Liying (1996) Analyse sur la gestion des résidents temporaires dans les villes et bourgs (Shilun woguo chengzhen zanzhu renkou de guanli), Population \& Economics, ${ }^{\circ}$ 4, pp. 3-9. 
WEI Zou (2003) Les métamorphoses des entreprises rurales, Perspectives chinoises, $\mathrm{n}^{\circ} 79$ septembre-octobre, pp. 18-31.

YANG Xiaoyong (1995) La migration des travailleurs et le développement économique dans les villes et campagnes (Mingong liudong yu zhongguo chengxiang jingji fazhan), Population \& Economics, $n^{\circ}$ 5, pp. 26-32.

YANG Yunyan (1992) Les changements de la migration en Chine dans les années 1980 (Bashi niandai zhongguo renkou qianyi de zhuanbian), Population \& Economics, $\mathrm{n}^{\circ}$ 5, pp. 12-16.

ZHANG Shenghua (1999) Shanghai Liudong Renkou de Zhanwang (Situtation et perspectives de la population migrante à Shanghai), Édition Université de la Chine de l'Est, 579 p.

ZHU Junyi, et YUAN Zhigang (2001) Shanghai jiuye baogao (Rapport sur les emplois à Shanghai), Shanghai renmin chubanshe, $262 \mathrm{p}$.

\section{NOTES}

1. Le capital spatial a été défini par J. Lévy et M. Lussault (2003) comme ensemble de ressources accumulées par un acteur, lui permettant de tirer avantage, en fonction de sa stratégie, de l'usage de la dimension spatiale de la société.

2. Cf. Recherche Insertions segmentées, discriminations et carrières dans l'emploi précaire des femmes provenant de Chine continentale, d'Europe de l'Est, d'Afrique subsaharienne et du Maghreb en France dirigée par L. Roulleau-Berger (en collaboration avec G. Giraudo, Ma Li, M. Lemaire et A. Tcholakova) dans le cadre de l'appel d'offres lancé en 2002 « La discrimination raciale dans l'emploi et sur le marché du travail» par la Direction de l'Animation de la recherche, des Études et Statistiques, Mission Animation de la Recherche du ministère de l'Emploi et de la Solidarité.

\section{RÉSUMÉS}

Cet article est centré sur l'analyse des migrations intracontinentales en Chine afin de comprendre comment se construisent les migrations transnationales, migrations liées entre elles par des réseaux économiques et sociaux fondés sur des relations de solidarité et d'entraide mutuelle. À partir des circulations et déplacements de migrants peu qualifiés on observera comment les espaces circulatoires en Chine ne cessent de se transformer, comment se diversifient des parcours, comment les destinations deviennent de plus en plus lointaines et comment se multiplient les lieux successifs d'installation quand la polymigration intracontinentale apparaît de plus en plus marquée et peut largement anticiper une migration transnationale. Enfin les migrations intracontinentales seront analysées comme pouvant produire des mobilités verticales ascendantes ou descendantes, entraînant ou non de la reconnaissance sociale dans la société chinoise contemporaine.

Migratory Routes and Circulations in China: between Intracontinental Mobilities and Transnationalism. This article focuses on the analysis of intracontinental migration in China in order to understand how transnational migration, linked together through economic and social networks based on solidarity and mutual assistance, is built up. By looking at the circulation and 
movement of poorly qualified migrants, we shall examine how zones of circulation in China are subject to constant transformation, how itineraries diversify, how destinations are becoming more and more remote, and how settlement locations increase one after another at a time when intracontinental polymigration seems to be ever more significant and can easily anticipate transnational migration. Finally, intracontinental migration will be analysed as potentially productive of upward or downward mobility, and of social recognition in contemporary Chinese society.

Rutas migratorias en China : entre movilidades intra-continentales y transnacionalismo. Este artículo se centra en el análisis de las migraciones intra-continentales en China. El objetivo es comprender cómo se construyen las migraciones transnacionales, migraciones ligadas entre sí por redes económicas y sociales fundadas en la solidaridad y la ayuda mutua. A partir de circulaciones y de desplazamientos de emigrantes poco calificados veremos cómo los espacios circulatorios no cesan de transformarse en China, cómo se diversifican los recorridos, cómo las destinaciones se vuelven cada vez más lejanas y cómo se multiplican los lugares de instalación en un momento en el que la poli-migración intra-continental gana en peso y anticipa, en numerosos casos, una migración transnacional. Las migraciones intra-continentales serán analizadas como productoras de movilidades verticales ascendentes o descendentes las cuales pueden o no derivar en un reconocimiento social por parte de la sociedad china contemporánea.

\section{INDEX}

Index géographique : Chine

Mots-clés : entraide, migrations intracontinentales, mobilité, réseaux sociaux

\section{AUTEURS}

\section{LAURENCE ROULLEAU-BERGER}

Sociologue, Chargée de recherches au CNRS, Habilitée à diriger des recherches, Groupe de recherche sur la socialisation (UMR 5040 CNRS), Université Lumière Lyon 2-ENS/LSH courriel : Laurence.Roulleau-Berger@univ-lyon2.fr

\section{LU SHI}

Maître de conférences, Département de chinois de l'Université Jean Moulin Lyon 3, Membre de l'Institut d'Asie Orientale. courriel : Lu.Shi@ens-lsh.fr 